\title{
Management of Giant Cervical Teratoma with Intracranial Extension Diagnosed in Utero
}

\author{
Jayesh P. Thawani ${ }^{1} \quad$ Michael J. Randazzo ${ }^{2} \quad$ Nickpreet Singh $^{2} \quad$ Jared M. Pisapia ${ }^{1} \quad$ Kalil G. Abdullah ${ }^{1}$ \\ Phillip B. Storm ${ }^{1,3,4}$
}

${ }^{1}$ Department of Neurosurgery, Hospital of the University of

Pennsylvania, Philadelphia, Pennsylvania, United States

2 Perelman School of Medicine, University of Pennsylvania,

Philadelphia, Pennsylvania, United States

${ }^{3}$ Division of Neurosurgery, Children's Hospital of Philadelphia,

Philadelphia, Pennsylvania, United States

${ }^{4}$ The Center for Fetal Diagnosis and Treatment, Children's Hospital of

Philadelphia, Philadelphia, Pennsylvania, United States

\author{
Address for correspondence Jayesh P. Thawani, MD, Department of \\ Neurosurgery, 3400 Spruce Street, Hospital of the University of \\ Pennsylvania, Philadelphia, PA 19104, United States \\ (e-mail: jayesh.thawani@uphs.upenn.edu).
}

J Neurol Surg Rep 2016;77:e118-e120.

\begin{abstract}
Keywords

- cervical teratoma

- EXIT procedure

- perinatal management

- polyhydramnios

Cervical teratomas are rare germ cell tumors affecting the fetus that are associated with significant morbidity and mortality due to an increased risk of airway obstruction at delivery. These tumors can commonly produce polyhydramnios that results from the fetus' impaired ability to swallow amniotic fluid. Improved rates of prenatal diagnosis through comprehensive evaluations and imaging have dramatically impacted the perinatal management of infants with this condition. Here, we report a patient diagnosed with polyhydramnios whose fetus was discovered to have a giant cervical teratoma on imaging studies. The child underwent surgical resection after having the airway secured under the uteroplacental support as part of an ex utero intrapartum treatment procedure performed at 37 weeks. The following gross pathological and magnetic resonance images demonstrate this condition and its currently accepted treatment.
\end{abstract}

\section{Introduction}

Cervical teratomas have an estimated incidence of 1 per 20,000 to 40,000 live births and represent the second most frequent perinatal germ cell tumor. ${ }^{1-3}$ Most of these tumors are benign, and therefore, complete resection at birth can be curative. However, the location of these tumors importantly can lead to airway distortion and obstruction. ${ }^{3}$ Furthermore, by compressing the hypopharynx and esophagus, giant teratomas can also prevent the swallowing of amniotic fluid resulting in polyhydramnios. Preterm labor, premature rupture of membranes, and placental abruption secondary to this polyhydramnios can complicate the surgical management of these tumors. ${ }^{4}$ As the incidence of polyhydramnios in previous large series has been reported as high as $82 \%$, this finding is critical not only for diagnosis but proper preoperative planning. ${ }^{1}$ This planning can be particularly useful in determining whether to proceed with a controlled delivery technique such as an ex utero intrapartum treatment (EXIT) procedure. We present a case whereby polyhydramnios on ultrasound prompted the discovery of an impressive lesion on maternal magnetic resonance (MR) imaging; we then describe the unique surgical treatment method that followed.

\section{Case Report}

A 32-year-old primigravid woman was diagnosed at 21 weeks gestation with polyhydramnios during routine screening. Her fetus was found to have a giant fetal neck mass by antenatal ultrasound. MR imaging was subsequently performed in
License terms

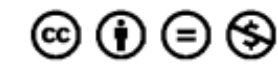

received

May 5, 2016

accepted

June 19, 2016
DOI http://dx.doi.org/

10.1055/s-0036-1586211. ISSN 2193-6358.

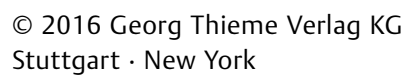


utero revealing a cystic and heterogeneous-appearing mass with extension into the middle and posterior cranial fossae consistent with a giant cervical teratoma. At 32 weeks gestation, imaging was repeated ( $\mathbf{- F i g . 1 A}$ ). Given the tumor's encroachment on the airway and esophagus, an EXIT procedure was planned at 37 weeks gestation to first secure the airway and then resect the mass following stabilization. 5,6

At the time of delivery, a cesarean delivery with the partial delivery of the fetus was undertaken. The child's head and upper body were exteriorized via hysterotomy guided by ultrasound. While attached to the placenta via the umbilical cord, anesthesia was administered. A combination of desflurane, propofol, and remifentanil were provided. ${ }^{7}$ An endotracheal tube was placed under direct laryngoscopy to secure the airway. Once secured, the umbilical cord was cut, and resuscitation was performed before surgical resection. The ensuing resection revealed that the tumor was extradural. Following a craniotomy, one portion of the tumor was resected from the middle fossa and was then followed through Kawase triangle into the posterior fossa. ${ }^{8}$ Enlargement and entry of the jugular foramen allowed for ligation of the jugular vein.

Grossly, the tumor was found to be protruding from the right ear canal and contained several pieces of abnormal bone (-Fig. 1B, C). These sections and the large cervical portion were removed en bloc. Pathology findings indicate a cystic teratoma containing scant immature tissue and more prominent mature tissue, consisting of skin, fibroadipose connective tissue, bone, cartilage, skeletal and smooth muscle, respiratory and gastrointestinal-type epithelium, pancreas-like glandular tissues, and lymphoid tissue. Postoperative MR imaging demonstrates near-total resection from the right middle cranial fossa, right posterior fossa, and right neck with little residual tumor (-Fig. 1D). Despite having a right-sided hearing deficit and swallowing dysfunction necessitating a gastrostomy tube,
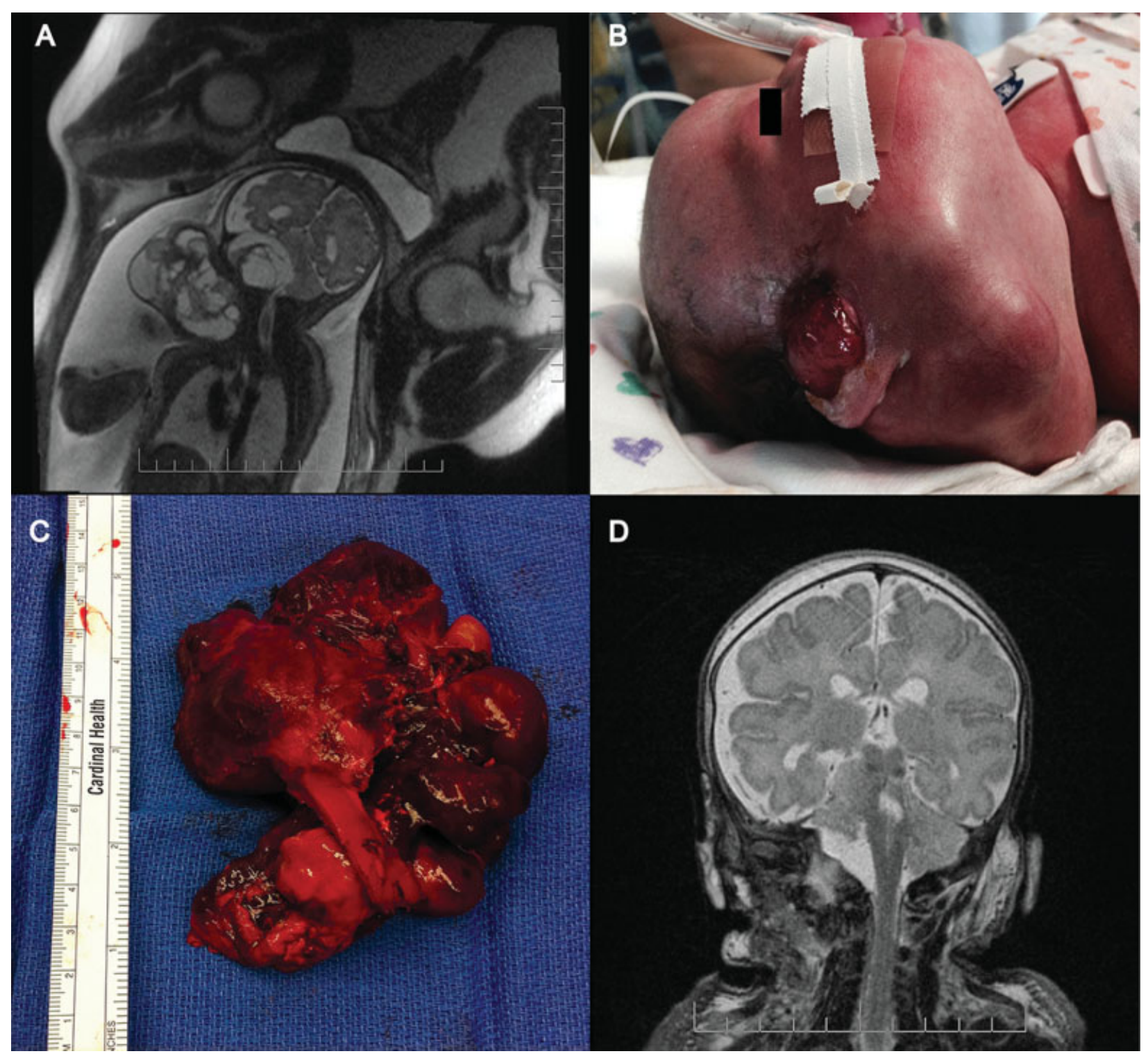

D

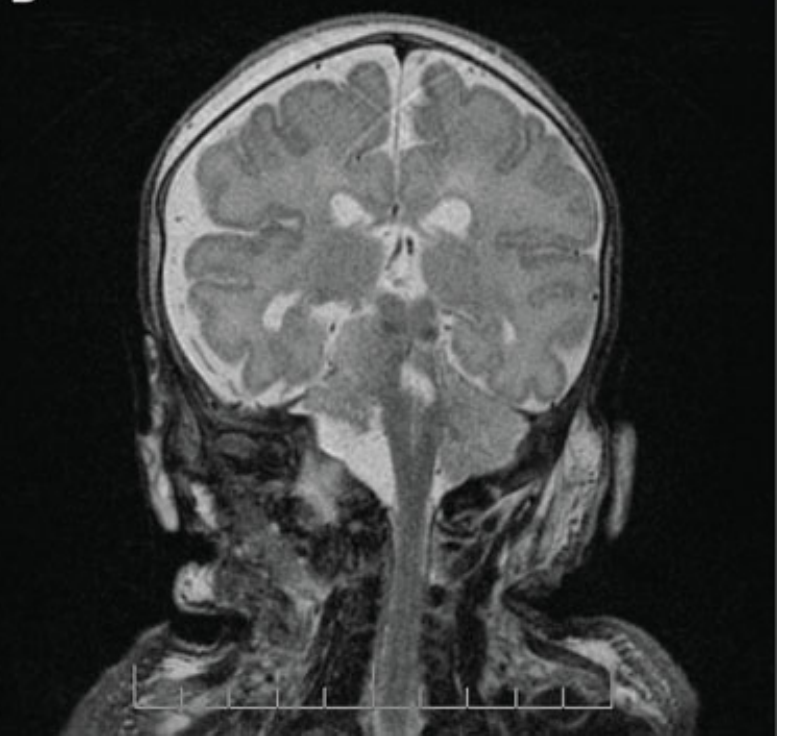

Fig. 1 (A) T2-weighted maternal MRI, 32 weeks gestation. A large fetal neck mass is seen with mass effect on the adjacent cerebellar hemisphere and brainstem. (B) Intraoperative view showing the mass protruding from the external ear. (C) Gross specimen with tissues including bone and hair. (D) Postoperative MRI at 3 months age. MRI, magnetic resonance imaging. 
at the time of this writing ( 6 months postoperatively), the child is breathing without a tracheostomy and meeting motor and cognitive milestones.

\section{Discussion}

Kelly et al first introduced the concept of operative management of a fetal cervical mass under uteroplacental support. ${ }^{9}$ The EXIT procedure was described and refined by Dr. Adzick, Dr. Harrison, and others in the 1990s. ${ }^{5,10,11}$ This fetal surgical procedure allows for safe delivery and the avoidance of airway obstruction, which carries with it a high risk of mortality in neonates. ${ }^{12}$ During the procedure, the head, neck, and shoulders of the fetus are delivered through a hysterotomy, thereby maintaining uterine volume and minimizing uterine contractions. Before the hysterotomy, intraoperative ultrasound is utilized to accurately map the placental edges to avoid placental abruption. Uteroplacental blood flow and gas exchange, as well as uterine relaxation, are also maintained through the use of inhalational agents, $\beta 2$ adrenergic agonists, and nitroglycerin. ${ }^{1}$ Movement of the fetus and breathing during the procedure is restricted to the use of vecuronium, atropine, and fentanyl, as previously described. ${ }^{5}$ In addition to the time needed for surgical resection, this provides adequate time for techniques necessary to secure the airway, including surfactant administration, direct laryngoscopy, bronchoscopy, and tracheostomy procedures. The EXIT procedure provides up to 1 hour of uteroplacental support in fetal patients with giant cervical teratomas. ${ }^{5}$ Possible complications relating to the EXIT procedure that were fortunately not encountered in this case include uterine atony, uterine rupture, and placental injury during hysterotomy. ${ }^{5}$

Previous reports of teratomas of the head and neck region have emphasized the importance of a skilled multidisciplinary team of physicians and nurses specializing in fetal, neonatal, and maternal care. ${ }^{1,5,13}$ Indeed, the rarity of these tumors and the expertise required to minimize complications necessitate careful planning and coordination to ensure the success of the EXIT procedure. Given the cervical and intracranial location of tumors such as that described, pediatric neurosurgeons remain integral to minimizing the morbidity associated with surgical treatment and in following patients through infancy and childhood.

\section{Conclusion}

Although rare, cervical teratomas can be diagnosed in the antenatal stage and treated safely using the EXIT procedure. Ultrasonography, by facilitating early diagnosis, MR imaging, by accurately illustrating anatomical relationships, and EXIT, by providing a mode of treatment, have all contributed complementary and beneficial roles in reducing mortality of cervical masses. This procedure allows for the partial delivery of the fetus to prevent airway compromise, a complication closely associated with infant mortality. Surgical resection of cervical teratomas in this manner can thus be used to minimize morbidity and mortality.

\section{Competing Interests}

None.

Funding

None.

\section{Acknowledgments}

The authors would like to acknowledge Yong Collins, NP for her assistance with obtaining the necessary medical records. No organizational funds were utilized in the design and conduct of the study or the collection, management, analysis, and interpretation of the data. All authors were responsible for the preparation, review, or approval of the article; and all are included in the decision to submit the article for publication.

\section{References}

1 Laje P, Johnson MP, Howell LJ, et al. Ex utero intrapartum treatment in the management of giant cervical teratomas. J Pediatr Surg 2012;47(6):1208-1216

2 Hubbard AM, Crombleholme TM, Adzick NS. Prenatal MRI evaluation of giant neck masses in preparation for the fetal exit procedure. Am J Perinatol 1998;15(4):253-257

3 Peiró JL, Sbragia L, Scorletti F, Lim FY, Shaaban A. Management of fetal teratomas. Pediatr Surg Int 2016

4 Sheikh F, Akinkuotu A, Olutoye OO, et al. Prenatally diagnosed neck masses: long-term outcomes and quality of life. J Pediatr Surg 2015;50(7):1210-1213

5 Liechty KW, Crombleholme TM, Flake AW, et al. Intrapartum airway management for giant fetal neck masses: the EXIT (ex utero intrapartum treatment) procedure. Am J Obstet Gynecol 1997;177(4):870-874

6 Jackson EM, Schwartz DM, Sestokas AK, et al. Intraoperative neurophysiological monitoring in patients undergoing tethered cord surgery after fetal myelomeningocele repair. J Neurosurg Pediatr 2014;13(4):355-361

7 Walz PC, Schroeder JW Jr. Prenatal diagnosis of obstructive head and neck masses and perinatal airway management: the ex utero intrapartum treatment procedure. Otolaryngol Clin North Am 2015;48(1):191-207

8 Kawase T, Toya S, Shiobara R, Mine T. Transpetrosal approach for aneurysms of the lower basilar artery. J Neurosurg 1985;63(6): 857-861

9 Kelly MF, Berenholz L, Rizzo KA, Greco R, Wolfson P, Zwillenberg DA. Approach for oxygenation of the newborn with airway obstruction due to a cervical mass. Ann Otol Rhinol Laryngol 1990;99(3 Pt 1):179-182

10 Mychaliska GB, Bealer JF, Graf JL, Rosen MA, Adzick NS, Harrison MR. Operating on placental support: the ex utero intrapartum treatment procedure. J Pediatr Surg 1997;32(2):227-230, discussion 230-231

11 Langer JC, Fitzgerald PG, Desa D, et al. Cervical cystic hygroma in the fetus: clinical spectrum and outcome. J Pediatr Surg 1990; 25(1):58-61, discussion 61-62

12 Azizkhan RG, Haase GM, Applebaum H, et al. Diagnosis, management, and outcome of cervicofacial teratomas in neonates: a Childrens Cancer Group study. J Pediatr Surg 1995;30(2):312-316

13 Taghavi K, Berkowitz RG, Fink AM, Farhadieh RD, Penington AJ. Perinatal airway management of neonatal cervical teratomas. Int J Pediatr Otorhinolaryngol 2012;76(7):1057-1060 